\title{
THE
}

\section{Critical new insights into the binding of poly- and perfluoroalkyl substances (PFAS) to albumin protein}

Jessica L. Alesio

Angela L. Slitt

University of Rhode Island, aslitt@uri.edu

Geoffrey D. Bothun

University of Rhode Island, gbothun@uri.edu

Follow this and additional works at: https://digitalcommons.uri.edu/che_facpubs

\section{The University of Rhode Island Faculty have made this article openly available.} Please let us know how Open Access to this research benefits you.

This is a pre-publication author manuscript of the final, published article.

Terms of Use

This article is made available under the terms and conditions applicable towards Open Access Policy Articles, as set forth in our Terms of Use.

\section{Citation/Publisher Attribution}

Alesio, J. L., Slitt, A., \& Bothun, G. D. (2022). Critical new insights into the binding of poly- and perfluoroalkyl substances (PFAS) to albumin protein. Chemosphere, 287(1), 131979. https://doi.org/ 10.1016/j.chemosphere.2021.131979

Available at: https://doi.org/10.1016/j.chemosphere.2021.131979 
1 Critical new insights into the binding of poly- and perfluoroalkyl substances (PFAS) to albumin protein

3 Kingston, Rhode Island 02881, United States.

10 *Corresponding Author: Geoffrey D. Bothun. E-mail: gbothun@uri.edu, Tel: +1-401-874-9518 


\section{ABSTRACT}

With an increasing number of health-related impacts of per- and polyfluoroalkyl

14 substances (PFAS) being reported, there is a pressing need to understand PFAS transport within

15 both the human body and the environment. As proteins can serve as a primary transport

16 mechanism for PFAS, understanding PFAS binding to proteins is essential for predictive

17 physiological models where accurate values of protein binding constants are vital. In this work

18 we present a critical analysis of three common models for analyzing PFAS binding to bovine

19 serum albumin (BSA) based on fluorescence quenching: the Stern-Volmer model, the modified

20 Stern-Volmer model, and the Hill equation. The PFAS examined include perfluorooctanoic acid

21 (PFOA), perfluorononanoic acid (PFNA), perfluorodecanoic acid (PFDA),

22 perfluorobutanesulfonic acid (PFBS), perfluorohexanesulfonic acid (PFHxS),

23 perfluorooctanesulfonic acid (PFOS), and the replacement compound 2,3,3,3-tetrafluoro-2-

24 (heptafluoropropoxy)propanoate (HFPO-DA or GenX). While all three models capture the 25 general effects of hydrophobicity and steric limitations to PFAS binding, the Hill equation

26 highlighted a unique relationship between binding cooperativity and the number of fluorinated

27 carbons, with PFOA exhibiting the greatest binding cooperativity. The significance of steric

28 limitations was confirmed by comparing results obtained by fluorescence quenching, which is an

29 indirect method based on specific binding, to those obtained by equilibrium dialysis where PFAS

30 binding directly correlated with traditional measures of hydrophobicity. Finally, the binding

31 constants were correlated with PFAS physicochemical properties where van der Waals volume

32 best described the steric limitations observed by fluorescence quenching.

33 Keywords: PFAS, protein binding, albumin, equilibrium dialysis, fluorescence quenching 


\section{INTRODUCTION}

Per- and polyfluoroalkyl substances (PFAS) are synthetic compounds produced for a

37 wide range of applications including nonstick industrial and commercial products, textiles, and

38 firefighting foams. Due to their persistence in the environment, human and ecosystem-related

39 exposures can occur long after release (Domingo and Nadal, 2017). Ninety-eight percent of the

40 United States population is expected to have detectable amounts of PFAS in their blood (Hu et

41 al., 2016). Water treatment plants are not equipped to remove all PFAS from water and thus,

42 drinking water creates another continuous exposure route (Ross et al., 2018). Although more

43 robust remediation systems are being created for the legacy compounds such as

44 perfluorooctanoic acid (PFOA) and perfluorooctanesulfonic acid (PFOS), shorter and longer

45 PFAS may not respond to the new treatment systems (Ahrens and Bundschuh, 2014; Xiao et al.,

46 2020). It is estimated that there are over 4,700 different PFAS and limited knowledge about even

47 the simplest of compounds (Boston et al., 2019). Once these PFAS enter the body, they are

48 linked to negative health effects such as immunosuppression, obesity, and insulin resistance

49 (Cardenas et al., 2017; Grandjean and Budtz-Jørgensen, 2013; Hartman et al., 2017)

50 As the scientific community discovers more PFAS, there is a need to understand the

51 effect on ecosystems and humans. Using bioaccumulation modeling, $\mathrm{Ng}$ and Hungerbühler

52 (2014) explained that both protein and lipid association are vital to the adaptation of models for

53 different PFAS. Dassuncao et al (2018) also used bioaccumulation modeling but were limited by

54 information available related to protein binding and lipid association. Another predictive tool

55 used is physiologically based pharmacokinetic/ pharmacodynamic (PBPK/PBPD) modeling but

56 also requires accurate tissue-protein partitioning coefficients. 
Due to the lack of PFAS protein binding data, researchers have used both computational models and laboratory experiments to obtain binding constants for different PFAS (Chen et al.,

59 2015; Chen and Guo, 2009; Cheng and Ng, 2018; Fedorenko et al., 2021; Qin et al., 2010;

60 Salvalaglio et al., 2010; Wu et al., 2009). Most of this work has been limited to legacy PFAS

61 (PFOA and PFOS). Bovine serum albumin protein (BSA) and/or the human equivalent (HSA)

62 are used because they are the most abundant in the blood and albumin has been shown to be the

63 major carrier of PFAS in the body (Forsthuber et al., 2020). In addition to transport, serum

64 protein binding of PFAS may provide insight into the effect of PFAS on the liver, as serum

65 albumin is catabolized in the liver and kidneys (Gitlin et al., 1958). A limited number of studies

66 have used equilibrium dialysis as a direct measure of the amount of PFAS bound to serum

67 albumin (Allendorf et al., 2019; Bischel et al., 2010; Gao et al., 2019; Wu et al., 2009). This

68 approach captures nonspecific interactions and specific binding events within the hydrophobic

69 pocket of serum albumin.

A common but indirect method to determine PFAS binding to serum albumin has been

71 fluorescence quenching (Chen et al., 2015; Chen and Guo, 2009; Hebert and MacManus-

72 Spencer, 2010; Macmanus-spencer et al., 2010; Qin et al., 2010; Wu et al., 2009). Fluorescence

73 quenching is a widely used spectroscopic technique that measures the binding of small organic

74 molecules and ions to proteins based on the intrinsic fluorescence of amino acid residues within

75 the protein structure. In bovine serum albumin (BSA, Figure S1), the fluorescence stems from

76 two tryptophan (Trp) residues, one located within the hydrophobic cavity of the protein (Trp-

77 213) and one closer the surface (Trp-134) and more accessible by the solvent (water) (Steinhardt

78 et al., 1971). The quenching behavior of these Trp residues indicates the extent of proximal

79 molecular binding and changes in the local polarity of the solvent environment, revealing 
80 information about the binding mechanism. Limitations to the fluorescence quenching method

81 include the assumptions behind the quenching models and the apparent lack of consensus as to

82 the appropriate ratio of PFAS to albumin.

83 In our previous work, Fedorenko et al. (2021) examined the entropic driving force for

84 PFAS binding to BSA using fluorine nuclear magnetic resonance spectroscopy $\left({ }^{19} \mathrm{~F}-\mathrm{NMR}\right)$ and

85 concluded that binding occurs via both specific binding within the hydrophobic pocket as well as

86 adsorption to the surface of the protein. Here, our aim was to examine how those different types

87 of binding events influence the fluorescence quenching of BSA and to evaluate the three

88 common models used to calculate PFAS-BSA association constants against equilibrium dialysis.

89 This work examined a range of perfluoroalkylcarboxylates ( $\mathrm{PFCA}, \mathrm{C}_{7, \mathrm{~F}}$ to $\mathrm{C}_{9, \mathrm{~F}}$ where $\mathrm{C}_{\mathrm{x}, \mathrm{F}}$

90 denotes the number of fluorinated carbons), perfluoroalkylsulfonates (PFSA; $\mathrm{C}_{4, \mathrm{~F}}, \mathrm{C}_{6, \mathrm{~F}}$ and $\mathrm{C}_{8, \mathrm{~F}}$ ),

91 and the replacement compound 2,3,3,3-tetrafluoro-2-(heptafluoropropoxy)propanoate (GenX;

$92 \mathrm{C}_{5, \mathrm{~F}}$; note that only three carbons are perfluorinated) and established binding relationships for

93 PFAS physicochemical properties (Scheme 1). Three different data analysis techniques were

94 used to correlate fluorescence quenching with protein binding: the Stern-Volmer model, the

95 modified Stern-Volmer model, and the Hill equation. Comparisons between fluorescence

96 quenching and equilibrium dialysis results provided key insights into the underlying

97 mechanisms. Relationships were established between octanol-water partitioning coefficients (log

$98 \mathrm{~K}_{\mathrm{ow}}$ ) as well as van der Waals volume and the extent of BSA binding to aid in prediction tools

99 for the wide range of PFAS. 

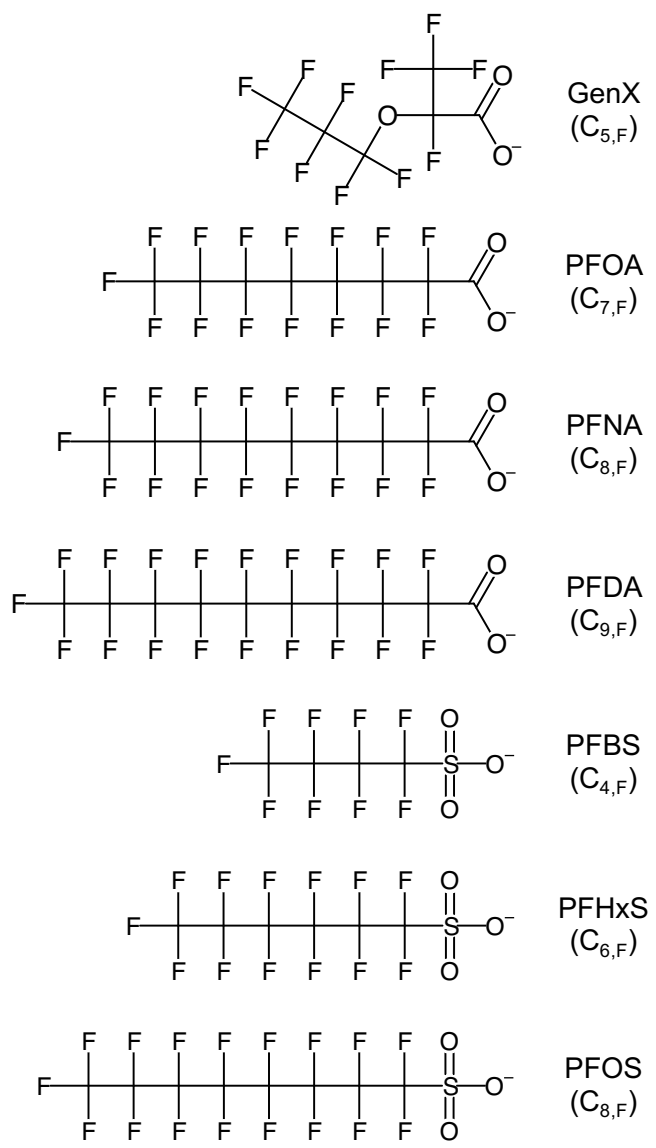

101 Scheme 1. PFAS examined. $\mathrm{C}_{\mathrm{x}, \mathrm{F}}$ denotes the number of fluorinated carbons.

102

103 MATERIALS AND METHODS

104 Materials. Bovine serum albumin (lyophilized powder, 99\% fatty acid free) was obtained from

105 Sigma-Aldrich (St. Louis, MO). A BSA concentration of $1.25 \mu \mathrm{M}$ in pH 7.4 phosphate buffered 106 saline (1X PBS) was used in each fluorescence quenching experiment. The solution of BSA was 107 kept at $4^{\circ} \mathrm{C}$ prior to use. Perfluorooctanoic acid, perfluorononanoic acid (PFNA), perfluorodecanoic acid (PFDA), 109 2,3,3,3-tetrafluoro-2-(heptafluoropropoxy)propanoate (Gen X), perfluorooctanesulfonic acid, 110 perfluorohexanesulfonic acid (PFHxS), and perfluorobutanesulfonic acid (PFBS) were obtained 
111 from Accustandard, Inc (New Haven, CT). PFAS solutions in PBS were stored at room

112 temperature in polypropylene containers.

113 Equilibrium Dialysis. Samples were prepared in a rapid equilibrium dialysis device (RED;

114 Thermo Fisher, Waltham, MA) such that the final concentrations of each mixture would contain

$11510 \mu \mathrm{M}$ bovine serum albumin (BSA) with either $10 \mu \mathrm{M}, 1 \mu \mathrm{M}$, or $20 \mu \mathrm{M}$ PFAS in phosphate

116 buffered saline (PBS). These samples were incubated for one hour at $37^{\circ} \mathrm{C}$ prior to

117 experimentation. The RED procedure was followed as described previously and as recommended

118 by the manufacturer (Gao et al., 2019; Waters et al., 2008). Controls of $10 \mu \mathrm{M}$ of each PFAS

119 were also added to the plate to account for PFAS adsorption onto the membrane or sides of the

120 chamber. After four hours of incubation at $37^{\circ} \mathrm{C}$ under constant shaking, samples were collected

121 from both sides. Samples from the buffer side, containing no protein, were prepared for analysis

122 by liquid chromatography/mass spectrometry (LC/MS). Details of the full LC/MS procedure and

123 association constant analysis can be found in the Supplementary Information.

124 The fraction of bound PFAS was determined by mass balance as $f=\left([\mathrm{PFAS}]_{\text {initial }}-\right.$

$\left.125[\mathrm{PFAS}]_{\text {unbound }}\right) /[\mathrm{PFAS}]_{\text {initial }}$ where $[\mathrm{PFAS}]_{\text {initial }}-[\mathrm{PFAS}]_{\text {unbound }}=[\mathrm{PFAS}]_{\text {bound. }}$ From $f$, the

126 protein/water partition coefficient, $K_{P W}\left(\mathrm{~g}\right.$ bound PFAS $\left.\mathrm{mL}^{-1} \mathrm{BSA}\right) /\left(\mathrm{g}\right.$ free PFAS mL ${ }^{-1}$ water $)$,

127 was determined as

128

$$
K_{P W}=\frac{f}{\rho_{P}[P](1-f)}
$$

129 where $\rho_{P}$ is the specific volume of the protein $\left(0.733 \mathrm{~mL} \mathrm{~g}^{-1}\right)$ and $[\mathrm{P}]$ is the total BSA

130 concentration $\left(10 \mu \mathrm{M}\right.$ or $\left.6.6 \times 10^{-3} \mathrm{~g} \mathrm{~mL}^{-1}\right)$ (Bischel et al., 2011). The association constant, $K_{a}\left(\mathrm{M}^{-}\right.$

$131{ }^{1}$ ), was calculated as (Allendorf et al., 2019)

132

$$
K_{a}=\frac{[P F A S]_{\text {bound }}}{\left([P]-[P F A S]_{\text {bound }}\right)[P F A S]_{\text {unbound }}}
$$


133 where the term $\left([\mathrm{P}]-[\mathrm{PFAS}]_{\text {bound }}\right)$ represents the residual unbound BSA protein assuming 1:1

134 molar binding.

135 Fluorescence Quenching. Quenching experiments were conducted using a PerkinElmer LS 55

136 Fluorescence Spectrophotometer with a $150 \mathrm{~W}$ xenon discharge lamp. The temperature was set 137 to $25^{\circ} \mathrm{C}, 30^{\circ} \mathrm{C}, 35^{\circ} \mathrm{C}$, or $40^{\circ} \mathrm{C}$ using a PerkinElmer PTP-1 Peltier Temperature Programmer. The 138 excitation wavelength was set to $295 \mathrm{~nm}$, data was collected from an emission range of $300 \mathrm{~nm}$ 139 to $500 \mathrm{~nm}$ at a rate of $100 \mathrm{~nm} / \mathrm{min}$, and the slit widths for both the emission and excitation were 140 set to $6 \mathrm{~nm}$.

141 A solution of BSA (2 mL) was added to a $1 \mathrm{~cm}$ quartz cuvette (Starna Cells, Atascadero, $142 \mathrm{CA}$ ) and the fluorescence spectrum of BSA alone was measured to provide a baseline. Aliquots 143 of PFAS stock solutions were added incrementally to achieve the desired concentration range. A 144 linear calibration curve of BSA fluorescence intensity over a range of concentrations was used to 145 correct for dilution of BSA. The quenching analyses performed herein were based on the 146 emission intensity at a wavelength of $349 \mathrm{~nm}$, which was the wavelength for maximum emission 147 intensity for BSA alone.

149 RESULTS AND DISCUSSION

150 PFAS binding by equilibrium dialysis

Equilibrium dialysis provides a direct measure of PFAS-protein binding through specific

152 and non-specific interactions, and a comparative basis for fluorescence quenching-based

153 association constants. The fraction of bound PFAS $f$ at $37^{\circ} \mathrm{C}$, the resulting protein/water partition

154 coefficient $K_{P W}$, and the calculated association constants $K_{a}$ are reported in Table 1 . In addition 
155 to representing physiological temperature, $37^{\circ} \mathrm{C}$ was used in accordance with reported protocols

156 for protein binding determined by equilibrium dialysis.

158 Table 1. Equilibrium dialysis results for PFAS-BSA binding at $37^{\circ} \mathrm{C}$.

\begin{tabular}{lcccc}
\hline PFAS & $\boldsymbol{f}^{\mathbf{a}}$ & $\boldsymbol{K}_{\boldsymbol{P W}}\left(\mathbf{1 0}^{\mathbf{5}}\right)^{\mathbf{a}}$ & $\boldsymbol{K}_{\boldsymbol{a}}\left(\mathbf{1 0}^{\mathbf{5}} \mathbf{M}^{-\mathbf{1}}\right)^{\mathbf{a}}$ & $\boldsymbol{K}_{\boldsymbol{a}}\left(\mathbf{1 0 ^ { 5 }} \boldsymbol{M}^{-\mathbf{1}}\right)^{\mathbf{b}}$ \\
\hline PFOA & $0.755(0.038)$ & $0.66(0.13)$ & $3.77(0.80)$ & $11(1.1)$ \\
PFNA & $0.975(0.005)$ & $8.52(1.62)$ & $44.05(8.37)$ & $14(2.2)$ \\
PFDA & $0.983(0.004)$ & $12.66(3.23)$ & $61.62(15.72)$ & $36(3.1)$ \\
PFBS $^{c}$ & $0.249(0.064)$ & $0.07(0.02)$ & $0.93(0.55)$ & $1.1(0.28)$ \\
PFHxS & $0.842(0.008)$ & $1.11(0.06)$ & $6.48(0.39)$ & $44(3.5)$ \\
PFOS & $0.942(0.004)$ & $3.39(0.27)$ & $17.99(1.44)$ & $32(4.9)$ \\
Gen $X^{c}$ & $0.265(0.006)$ & $0.07(0.002)$ & $0.65(0.04)$ & $0.23(0.05)$ \\
\hline
\end{tabular}

159

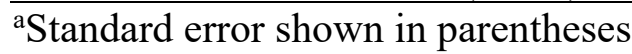

$160 \quad{ }^{b}$ Reported by Allendorf et al. (2019)

$161{ }^{\mathrm{c}} K_{P W}$ and $K_{a}$ determined at a PFAS:BSA molar ratio of 2:1. All other values are determined at a

162 0.1:1 molar ratio (Additional details in the Supplemental Information).

163

164

The values for the fraction of bound PFAS, $f$, and the resulting $K_{P W}$ values obtained in

165 this work are in agreement with Bischel et al (2011) for the longer compounds (PFNA, PFDA,

166 and PFOS) but not for the shorter compounds where $f$ values $\geq 0.99$ were reported for PFOA,

167 PFBS, and PFHxS. The $f$ values for these shorter compounds fall between those reported by

168 Bischel et al. (2011) and Allendorf et al. (2019), where low bound fractions were observed for

169 PFOA and PFBS $(f=0.44)$, and PFHxS $(f=0.62)$. In this work, $f$ increased with the number of

170 fluorinated carbons from $\mathrm{C}_{4, \mathrm{~F}}$ to $\mathrm{C}_{9, \mathrm{~F}}$. There was no apparent evidence for longer compounds

171 adopting a helical structure that would result in maximum $K_{P W}$ values at $\mathrm{C}_{6, \mathrm{~F}}$ with decreases in

$172 K_{P W}$ above $\mathrm{C}_{7, \mathrm{~F}}$ (Bischel et al., 2011). 
173 A positive correlation was observed between the association constants and the logarithm

174 of the octanol-water partition coefficient, $\log \mathrm{K}_{\mathrm{ow}}$, for non-ionic (associated; obtained from

175 Pubmed; Figure 1A) and ionic (dissociated at pH 7.4; calculated using Marvin Sketch; Figure

176 1B) PFAS species, in agreement with values reported previously using PFAS chain length as a

177 proxy for hydrophobicity (Table 1) (Allendorf et al., 2019). The PFCA correlation includes

178 GenX, suggesting that binding of this alternative PFAS based on hydrophobicity is similar to the

179 linear PFCA. Log $\mathrm{K}_{\mathrm{ow}}$ correlations are used to examine quantitative structure-activity

180 relationships (QSAR) governing ligand binding to proteins, with the $\log \mathrm{K}_{\mathrm{ow}}$ coefficient

181 reflecting the strength of hydrophobic binding and depth of insertion into the protein structure

182 (Hansch and Klein, 1986). A coefficient $\geq 1$ indicates a strong dependence on hydrophobicity

183 and deep insertion upon binding, which was observed for PFCA and PFSA. Larger log $\mathrm{K}_{\mathrm{ow}}$

184 coefficients were determined for PFCA, suggesting a stronger dependence on hydrophobicity. 

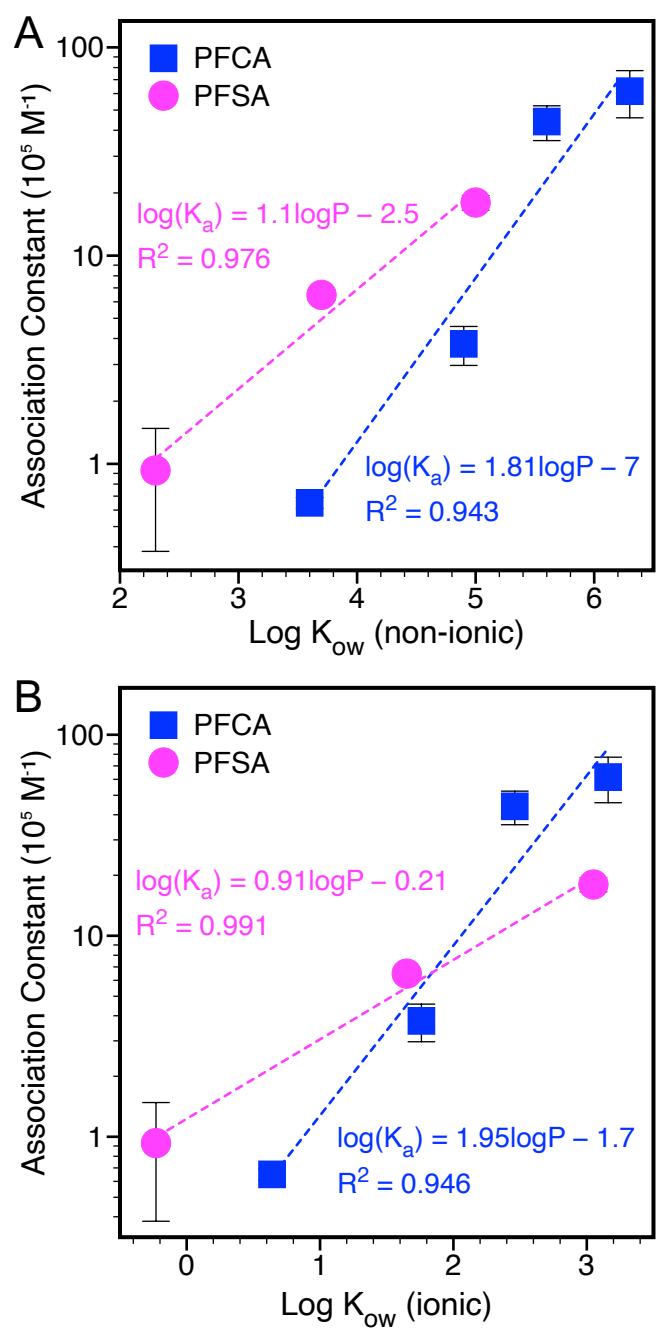

186 Figure 1. PFAS-BSA association constant determined by equilibrium dialysis as a function of the

187 logarithm of the octanol-water partition coefficient, $\log \mathrm{K}_{\mathrm{ow}}$, for (A) non-ionic (associated;

188 obtained from Pubmed) and (B) ionic (dissociated at pH 7.4; calculated using Marvin Sketch)

189 PFAS species. Standard error bars not observed are smaller than the symbols. $K_{a}$ in the fitted 190 equations has units of $10^{5} \mathrm{M}^{-1}$.

\section{PFAS binding by fluorescence quenching}

In contrast to equilibrium dialysis measurements, fluorescence quenching-based

194 measurements are based on PFAS binding to specific sites on BSA containing tryptophan (Trp) 
195 residues. The extent of fluorescence quenching can be directly related to PFAS concentration

196 through the Stern-Volmer equation, which has been reported for PFAS-BSA binding (Bischel et

197 al., 2011, 2010; Chen et al., 2015; MacManus-Spencer et al., 2010; Qin et al., 2010). The Stern-

198 Volmer equation is written as (Lakowicz, 2006)

$$
\frac{F_{0}}{F}-1=K_{S V}[Q]
$$

200 where $F$ is the fluorescence emission intensity at a specified wavelength $(349 \mathrm{~nm})$ of the protein

201 in the presence of the quencher, $F_{0}$ is the fluorescence emission intensity of the protein in the

202 absence of the quencher, $K_{S V}$ is the Stern-Volmer association constant, and $[Q]$ is the

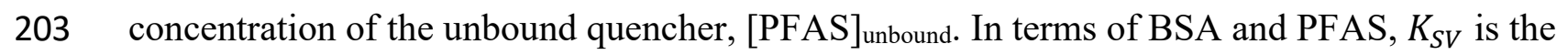

204 degree of association between the quencher and the protein at equilibrium

205

206

$$
\begin{gathered}
{[P F A S]+[B S A] \rightleftharpoons[B S A-P F A S]} \\
K_{S V} \approx K_{e q}=\frac{[B S A-P F A S]}{[P F A S][B S A]}
\end{gathered}
$$

207 where [BSA-PFAS] is the concentration of the bound complex. The Stern-Volmer equation is 208 based on the following assumptions (Eftink and Ghiron, 1981; Lakowicz, 2006): (i) The

209 concentration of the quencher is large relative to protein concentration such that $[Q]_{\text {total }}$ (or

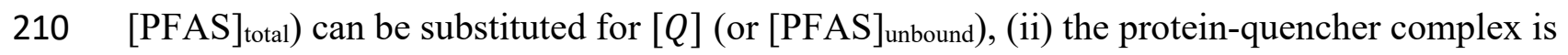
211 nonfluorescent, and (iii) binding is noncooperative.

212 Exemplary spectra and Stern-Volmer plots are shown in the Figures S2 and S3,

213 respectively. With the exception of PFNA, equation 3 yielded a linear curve suggesting a static

214 quenching process where the BSA-PFAS complex is non-fluorescent. However, fitted $K_{S V}$ values 215 over [PFAS] from 0 to $6.8 \mu \mathrm{M}$ did not change appreciably over the temperature range examined 216 (Figure 2A; Table S2), in contrast with reported studies showing a strong temperature 
217 dependence indicative of enthalpic binding. This may reflect a more dominant entropic

218 contribution as previously shown using ${ }^{19} \mathrm{~F}-\mathrm{NMR}$ (Fedorenko et al., 2021) or an apparent

219 combined static and dynamic quenching process, where elevated temperatures increase the

220 diffusivity of binding ligands and thus the collision frequency with the binding site. Compared to

221 previous studies, the $K_{S V}$ values obtained in this work at $25^{\circ} \mathrm{C}$ are on the same order of

222 magnitude for PFOA and PFOS at reported temperatures ranging from $21^{\circ} \mathrm{C}$ to $27^{\circ} \mathrm{C}$ (Chen et al.,

223 2015; Li et al., 2010; Qin et al., 2010).

224
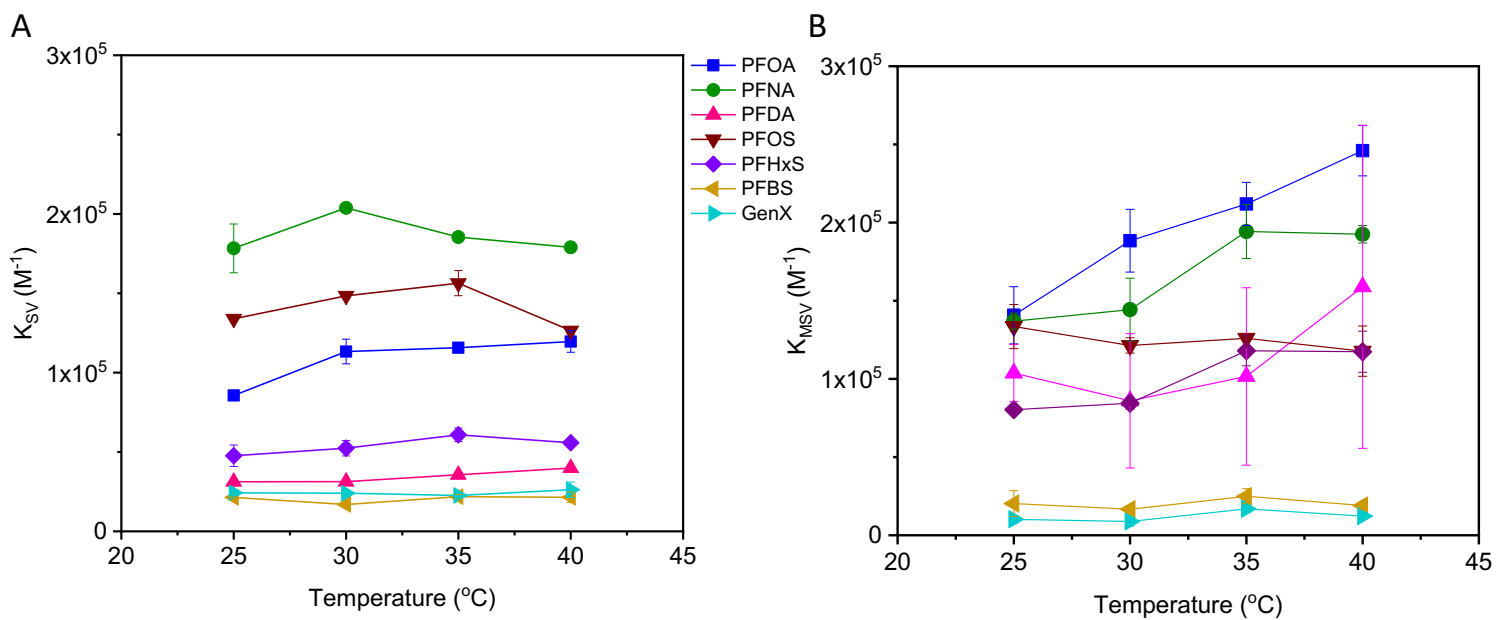

225

Figure 2. A) Stern-Volmer $\left(K_{S V}\right.$; equation 3, Figure S3) and B) modified Stern-Volmer $\left(K_{M S V}\right.$;

227 equation 6, Figure S4) association constants for PFAS binding to BSA as a function of

228 temperature. Standard error bars shown are based on two independent measurements.

$231[\mathrm{PFAS}]_{\text {unbound. }}$ To test this assumption, $[\mathrm{PFAS}]_{\text {total }}$ was increased ten-fold and $[\mathrm{BSA}]$ was held

232 constant. Results for fluorescence quenching decayed exponentially with increasing [PFAS],

233 displaying a plateau at high [PFAS]. In this case, the modified Stern-Volmer equation was 
234 required to account for saturation by incorporating a fractional accessibility term, $f_{a}$, of the 235 PFAS to the quenching site (Chen and Guo, 2009; Qin et al., 2010)

$$
\frac{F_{0}}{F_{0}-F}=\frac{1}{f_{a} K_{M S V}[Q]}+\frac{1}{f_{a}}
$$

237 where $K_{M S V}$ is the modified Stern-Volmer association constant. Equation 6 accounts for 238 downward curvature in Stern-Volmer plots and assumes that, as [Q] $\rightarrow \infty$, only the inaccessible

239 fluorescent amino acid residues can fluoresce. Thus, it allows for two types of fluorescent

240 residues in a protein - those accessible to the quencher and those not accessible to the

241 quencher - with no allowance for a partially accessible fluorophore or for binding cooperativity

242 (Lakowicz, 2006). For BSA this involves PFAS binding near Trp-214 and Trp-134, with one of

243 these sites being inaccessible. The modified Stern-Volmer equation was applied over [PFAS]

244 ranging from 14.7 to $68.2 \mu \mathrm{M}$ for PFCAs and 7.4 to $74.2 \mu \mathrm{M}$ or 35.7 to $74.2 \mu \mathrm{M}$ for PFSAs.

245 Data obtained below these lower [PFAS] limits led to poor fits.

246 Fitted $K_{M S V}$ were similar to $K_{S V}$ for many of the PFAS examined (Figure 2, Tables S2

247 and $\mathrm{S} 3$ ), which implies that the assumption $[\mathrm{PFAS}]_{\text {total }} \approx[\mathrm{PFAS}]_{\text {unbound }}$ is reasonable for the

248 Stern-Volmer model and that 'fractional accessibility' accounts for saturation at high [PFAS] in

249 the modified Stern-Volmer model. However, the trends in the association constants with PFAS

250 size were different with the exception of the two smallest PFAS, GenX and PFBS. These trends

251 are shown below for $25^{\circ} \mathrm{C}$ with the largest PFAS, PFDA, in bold and the sulfonates underlined to

252 distinguish them.

253

254

255

256 exception ( 0.3). Mechanistically, this means that the binding sites with fluorescent Trp residues 
257 are less accessible to PFBS binding. Being the smallest and most water soluble PFAS examined,

258 PFBS $\left(\mathrm{C}_{\mathrm{n}, \mathrm{F}}=4\right)$ may have lacked the hydrophobicity needed to insert into the hydrophobic,

259 tryptophan-containing regions of BSA. Interestingly, this argument does not apply to $\mathrm{GenX}\left(\mathrm{C}_{\mathrm{n}, \mathrm{F}}\right.$

$260=5$ ), which showed similarly small $K_{M S V}$ values with $f_{a}$ values that were two-fold higher than

261 PFBS. Despite GenX having access to the binding sites, the affinity between GenX and the

262 binding site was low. This may be due to the non-linear structure where the $\mathrm{CF}_{3}$ group near the

263 carboxylic acid headgroup sterically hindered binding.

264 The third model applied to the fluorescence quenching data - the Hill equation - also

265 accounts for the residual fluorescence upon saturation, $F_{r e s}$, but does not ascribe $F_{\text {res }}$ to

266 fractional accessibility. The model applied is based on the Hill approximation proposed by

267 Hebert et al for PFAS-protein binding (Hebert and MacManus-Spencer, 2010)

$$
\frac{F_{0}-F}{F_{0}-F_{\text {res }}}=\left[\frac{\left(K_{\text {Hill }}[Q]\right)^{n_{\text {Hill }}}}{1+\left(K_{\text {Hill }}[Q]\right)^{n_{\text {Hill }}}}\right]
$$

269 where $K_{\text {Hill }}$ and $n_{\text {Hill }}$ are the Hill binding constant and coefficient, respectively. At high [PFAS]

270 relative to [BSA], this model accurately reflects the quenching conditions, which are dependent

271 upon the change in protein conformation with the type and concentration of PFAS (Hebert and

272 MacManus-Spencer, 2010). This dependence is described by $n_{\text {Hill }}$, which accounts for binding

273 cooperativity. Hill coefficients greater than $1, n_{\text {Hill }}>1$, denote positive binding cooperativity,

$274 n_{\text {Hill }}<1$ denote negative binding cooperativity, and $n_{\text {Hill }}=1$ denote noncooperative binding.

275 Unlike the Stern-Volmer and modified Stern-Volmer models where data sets were truncated to

276 accommodate the models, excellent fits were obtained for the Hill model over the entire

277 concentration range examined.

278 Comparisons between $K_{H i l l}, K_{M S V}$ and $K_{S V}$ are shown in Figure 3 at the four temperatures 279 examined (data presented in Table S3). Overall, the association constants based on $K_{\text {Hill }}$ were 
280 similar to or greater than that for $K_{M S V}$ and $K_{S V}$; or alternatively, the Stern-Volmer models

281 underestimated PFAS-BSA binding compared to the Hill model. This was particularly true for

282 the most hydrophobic carboxylic acids, PFOA, PFNA, and PFDA. There was comparatively

283 better agreement between $K_{H i l l}, K_{M S V}$ and $K_{S V}$ with the sulfonic acids.
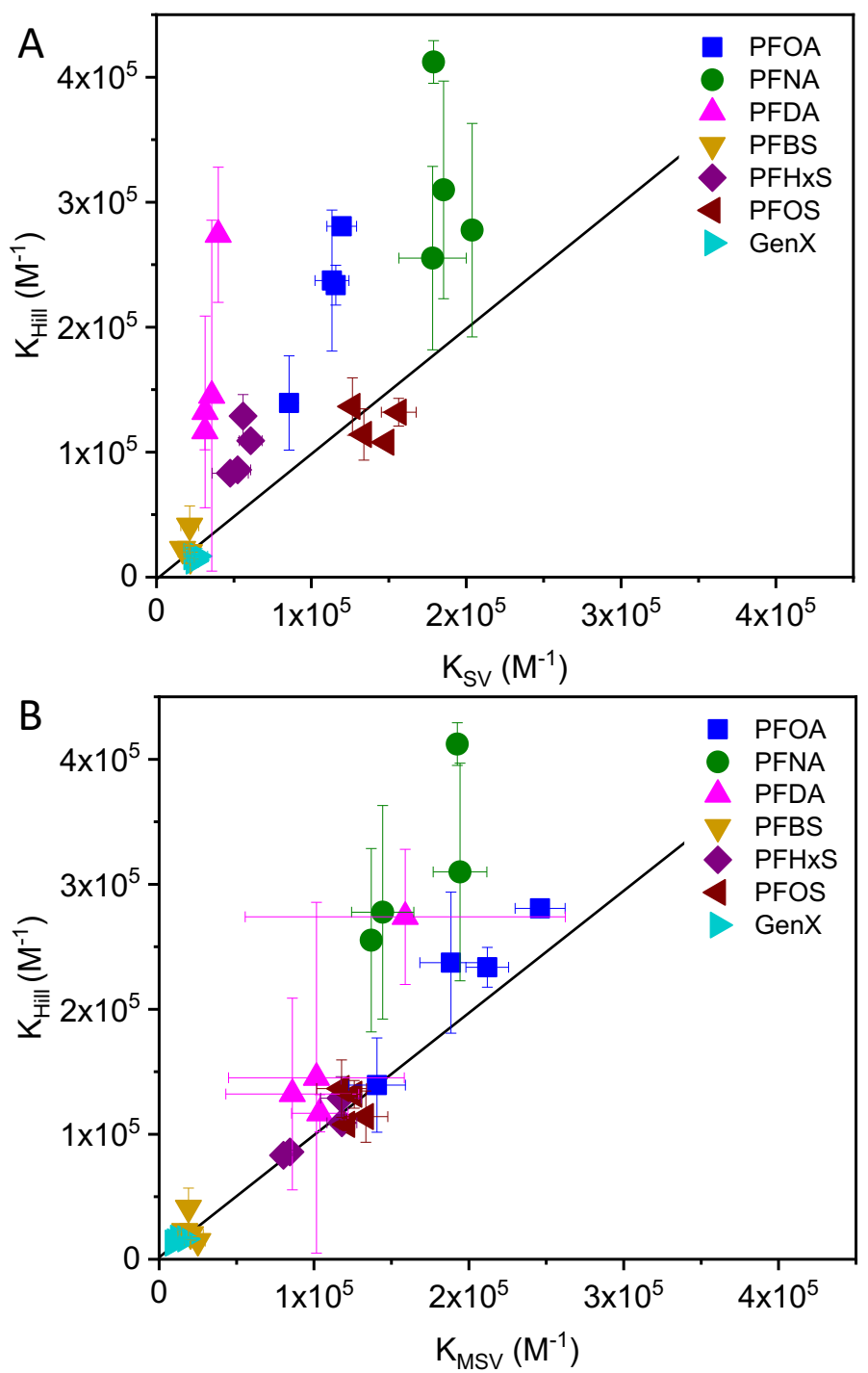

Figure 3. Comparison of PFAS-BSA association constants determined by fluorescence

286 quenching from the Hill equation $\left(K_{H i l l}\right)$ to those determined using the (A) Stern-Volmer $\left(K_{S V}\right)$ 287 and (B) modified Stern-Volmer $\left(K_{M S V}\right)$ equations. The data points for each PFAS were obtained 
288 at temperatures of $25^{\circ} \mathrm{C}, 30^{\circ} \mathrm{C}, 35^{\circ} \mathrm{C}$, and $40^{\circ} \mathrm{C}$ (data shown in Table S3). The solid lines

289 represent the condition where the association constants are equal.

290

291 Additional insight can be gained by analyzing the binding cooperativity described by

$292 n_{\text {Hill }}$ as a function of the number of fluorinated carbons (Figure 4). Results shown at $25^{\circ} \mathrm{C}$ and

$29335^{\circ} \mathrm{C}$ depict two regions - negative cooperativity or noncooperative/positive cooperativity - that

294 are independent of temperature. Negative binding cooperativity $\left(n_{\text {Hill }}<1\right)$ was observed for

295 small (PFBS, $\left.C_{n, F}=4\right)$ and large (PFDA, $\left.C_{n, F}=9\right)$ PFAS, while noncooperative (GenX, PFNA)

296 or positive binding cooperativity (PFHxS, PFOA, PFOS) was observed for PFAS with $\mathrm{C}_{\mathrm{n}, \mathrm{F}}=5$ to

$2978\left(n_{H i l l} \geq 1\right)$. The trend in binding cooperativity with $\mathrm{C}_{\mathrm{n}, \mathrm{F}}$ may reflect the ability for intermediate

298 PFAS to 'fit within' and bind to the hydrophobic regions containing Trp, leading to

299 conformational changes within the protein that further increases binding affinity (Bischel et al.,

300 2011).

301

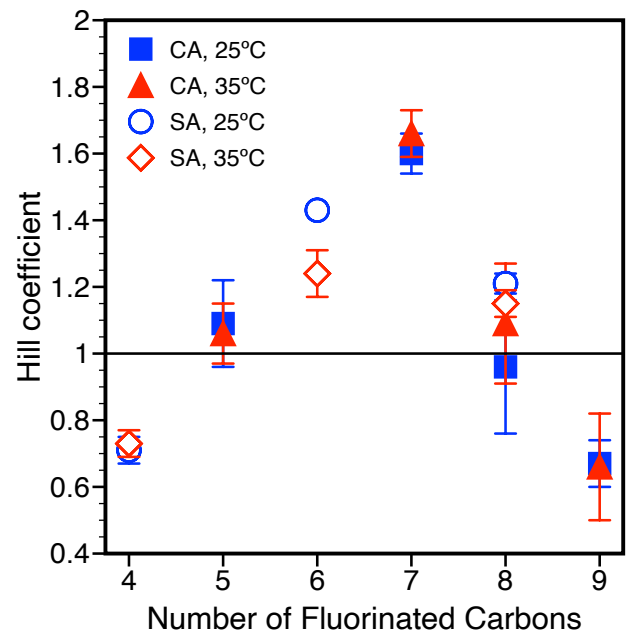

302 Figure 4. Hill coefficient, obtained from the Hill model fitting (equation 7), describing PFAS-

303 BSA binding cooperativity. Standard error bars shown are based on three independent 
measurements for PFCAs and two independent measurements for PFSAs. PFCA and PFSA are

305 abbreviated as CA and SA, respectively.

To further describe the trends in binding across the range of PFAS, the association

308 constants obtained via fluorescence quenching were compared to molecular descriptors. Results

309 for $K_{a}$ versus the number of fluorinated carbons (Figure 5A) agree with previous work showing 310 that PFOA and PFOS preferentially bind to a hydrophobic cavity within a serum albumin protein

311 (Chen and Guo, 2009; Salvalaglio et al., 2010). However, the number of fluorinated carbons is

312 not an adequate proxy for molecular size. PFNA showed the highest association constant, higher

313 than PFOS, despite having the same number of fluorinated carbons. An analysis of the van der

314 Waals volume, $V_{\mathrm{vdW}}$, shows that PFOS $\left(\mathrm{C}_{8, \mathrm{~F}}\right)$ is larger than PFNA $\left(\mathrm{C}_{8, \mathrm{~F}}\right)$ and similar in size to

315 PFDA $\left(\mathrm{C}_{9, \mathrm{~F}}\right)$, which also showed a lower $K_{a}$ compared to PFNA (Figure 5B). The importance of

316 steric hinderance is further denoted by PFOA $\left(\mathrm{C}_{7, \mathrm{~F}}\right)$, which is less hydrophobic than PFOS and

317 has a considerably smaller $V_{\mathrm{vdW}}$, but similar association with BSA. The trends in $K_{a}$ with $V_{\mathrm{vdW}}$

318 are similar to those with the ionic $\log \mathrm{K}_{\mathrm{ow}}$ (Figure 5C), which takes into consideration the

319 headgroup charge, where the two largest PFAS, PFOS and PFDA, are also the most hydrophobic

320 yet exhibit a lower $K_{a}$ than PFNA. This trend was not captured with the associated $\log \mathrm{K}_{\mathrm{ow}}$

321 values (Figure 5D). Above an optimal size, described by $V_{\mathrm{vdW}}$, the PFAS molecules no longer

322 'fit' into the same location on BSA regardless of their hydrophobicity. 

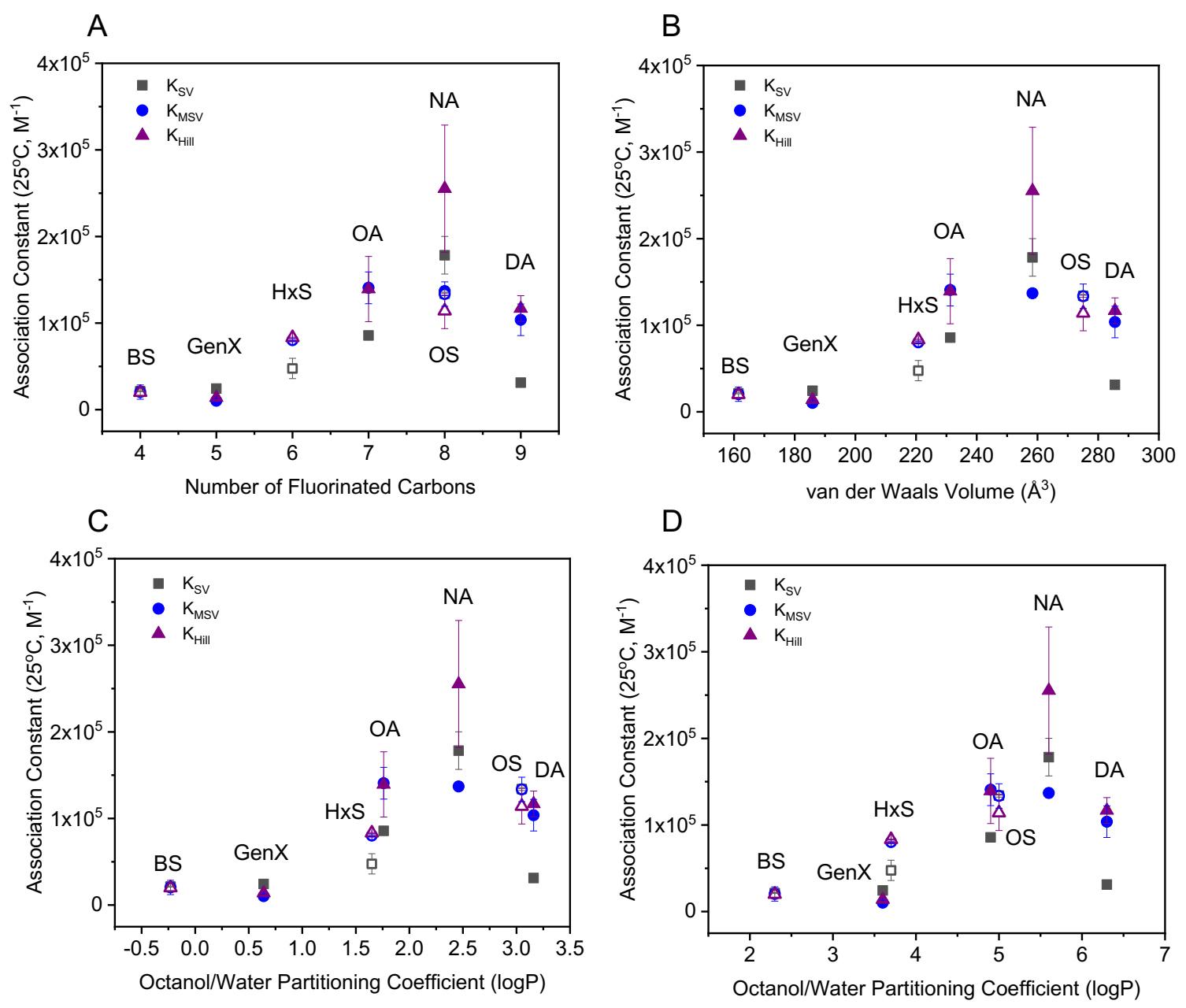

324 Figure 5. PFAS association constants determined by fluorescence quenching as a function of

325 molecular descriptors: A) the number of fluorinated carbons, B) the calculated van der Waals

326 volume, C) the logarithm of the ionic the octanol-water partitioning coefficient, or ionic log $\mathrm{K}_{\mathrm{ow}}$,

327 calculated at $\mathrm{pH} 7.4$, and D) the calculated logarithm of the octanol-water partitioning coefficient

328 or $\log \mathrm{K}_{\text {ow. }}$. Closed symbols correspond with PFCA and open symbols PFSA.

329

The hydrophobic nature of the PFAS-protein interactions can be explored further by

331 evaluating the wavelength shift in the fluorescence spectra (Figure S5), consistent with previous

332 studies of both BSA and HSA (Liu et al., 2017; Qin et al., 2010; Wu et al., 2009). A shift in the 
333 wavelength associated with maximum fluorescence emission indicates a change in the solvent

334 environment surrounding the fluorescent Trp residues. PFBS and GenX do not show marked

335 shifts in emission wavelength, consistent with low binding affinity within the hydrophobic cavity

336 of BSA and/or their inability to dehydrate the cavity and cause local decreases in solvent

337 polarity. For all other PFAS, the blue shift indicates that PFAS binding created a more

338 hydrophobic environment as the conformation of the protein changes and bound water molecules 339 are displaced by the PFAS (Vivian and Callis, 2001). The extent of wavelength shift roughly 340 correlates with PFAS hydrophobicity. The maximum shifts occur for PFNA ( $-13 \mathrm{~nm})$ for the 341 carboxylates and PFHxS $(-10 \mathrm{~nm})$ for the sulfonates. The shift was not as great for the larger 342 PFDA $(-10 \mathrm{~nm})$ or PFOS $(-7 \mathrm{~nm})$, supporting the concept that these molecules do not fit as well

343 within the cavity, which has been reported for PFDA but not PFOS (Bischel et al., 2010;

344 Macmanus-spencer et al., 2010).

346 Mechanistic insight comparing fluorescence quenching and equilibrium dialysis

347 The difference between the two experimental methods presented here is that fluorescence 348 quenching reflects PFAS binding within the hydrophobic cavity of BSA, while equilibrium 349 dialysis reflects all binding. Comparing these results can shed more light on the interaction of 350 different PFAS with BSA. For PFDA (Figure 6), the $K_{a}$ from equilibrium dialysis is an order of 351 magnitude higher than $K_{a}$ values determined by fluorescence quenching. As PFDA is the largest 352 molecule studied (Figure 5B) based on $V_{\mathrm{vdw}}$, size exclusion from the hydrophobic pockets may 353 make binding to the surface more energetically favorable. For sulfonates, PFOS shows a similar 354 trend. Although PFOS and PFNA have the same number of fluorinated carbons, PFNA has a 355 higher association constant than PFOS. 


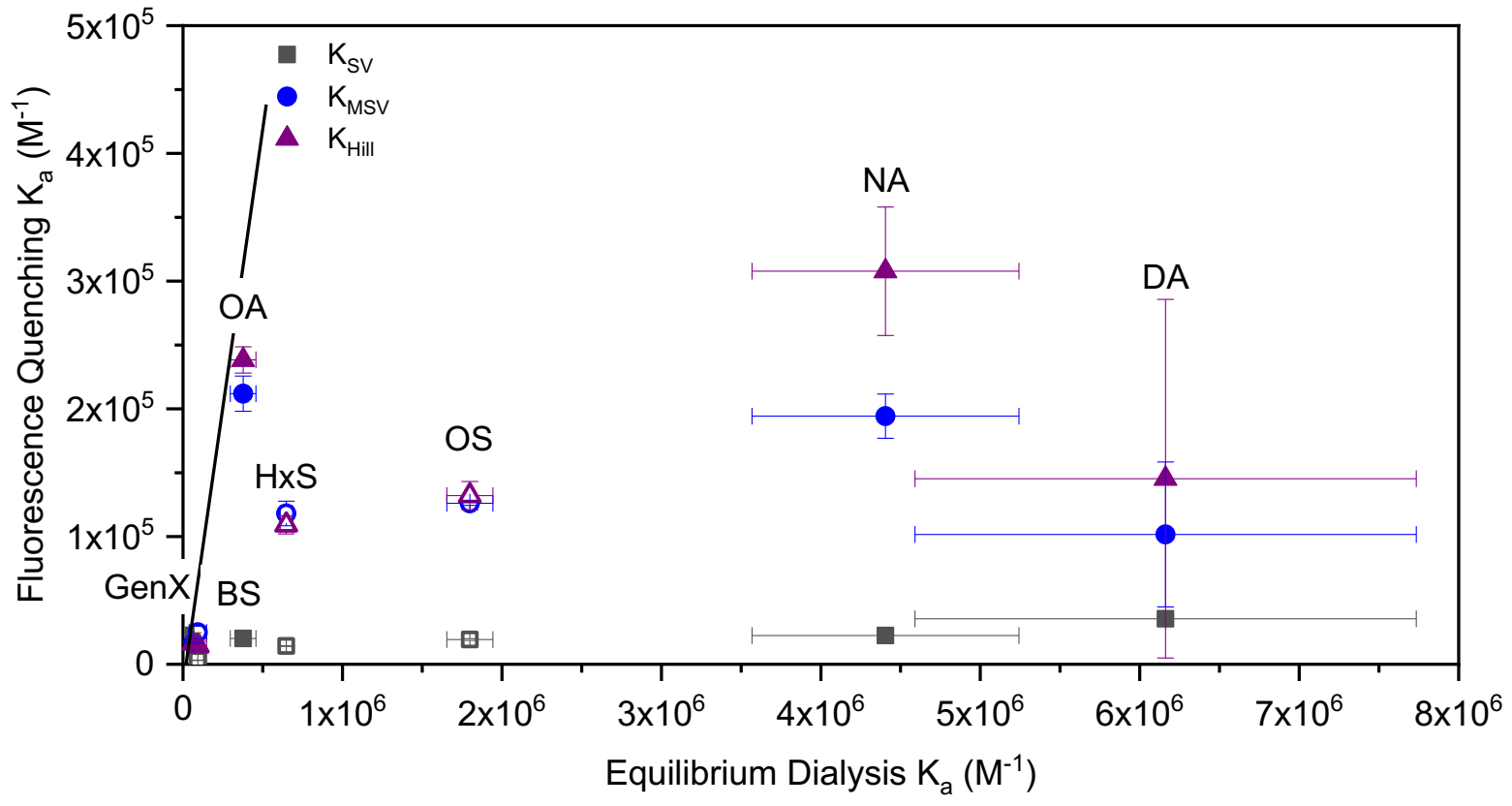

358 Figure 6. A comparison of association constants determined at $35^{\circ} \mathrm{C}$ by fluorescence quenching 359 and equilibrium dialysis. The solid lines represent the condition where the association constants are equal. Closed symbols correspond with PFCA and open symbols PFSA.

363 accounts for binding cooperativity, is in closer agreement with the 'true' extent of binding based 364 on equilibrium dialysis, particularly for the more hydrophobic PFAS. However, the results 365 clearly demonstrate that both approaches should be combined to gain a mechanistic 366 understanding of PFAS binding as a function of their physicochemical properties.

367 The importance of understanding the effect of physicochemical properties on protein 368 binding becomes more apparent as industry producers move toward using shorter chain length 369 PFAS. However, shorter chains do not necessarily result in lower protein binding affinities than 370 longer chain PFAS. Our work shows, based on both experimental techniques, that PFHxS binds 
371 to BSA with similar affinity as PFOS. This is significant to exposure assessment and health risks

372 as albumin has been shown to be the major carrier of PFAS in human blood (Forsthuber et al.,

373 2020). As more PFASs are developed as alternatives to legacy compounds, there is a pressing

374 need to continue to rigorously assess protein binding and identify correlations with PFAS

375 molecular properties. One way to accomplish this is through the link between $\log \mathrm{K}_{\mathrm{ow}}$ and degree

376 of binding. For example, as $\log \mathrm{K}_{\mathrm{ow}}$ (hydrophobicity) increases for the PFAS of $\mathrm{C}_{8, \mathrm{~F}}$ and smaller

377 in this study, the degree of binding to BSA increases. As is shown with PFDA $\left(\mathrm{C}_{9, \mathrm{~F}}\right)$, this model

378 parameter should be coupled with Van der Waals volume to account for steric hinderance to

379 binding.

380

\section{CONCLUSIONS AND IMPLICATIONS}

382

With albumin being a major protein carrier for PFAS in vivo, accurate protein binding

383 constants and mechanistic insight into binding are imperative to guide bioaccumulation models

384 and physiologically based pharmacokinetic/pharmacodynamic (PBPK/PBPD) models. In this

385 work, a systematic analysis of fluorescence-based protein binding models was conducted for an

386 expanded range of PFAS and compared to equilibrium dialysis experiments. Both experimental

387 methods provide valuable and complimentary insight into PFAS- BSA binding. While

388 fluorescence quenching focuses on the hydrophobic pocket of the protein, equilibrium dialysis

389 reports all types of binding, whether in specific locations or to the surface. By critically

390 examining the differences between the equilibrium dialysis and fluorescence quenching, we can

391 gain insight into binding mechanisms, especially for those PFAS-serum protein interactions that

392 have not yet been characterized by crystal structure or other methods. 
Fluorescence quenching has been used to evaluate PFAS binding to serum albumin

394 proteins but there is no clear guidance on the applicability or limitations of the three models

395 discussed in this work: the Stern-Volmer model, the modified Stern-Volmer model, and the Hill

396 equation. By limiting analysis to one of these methods, valuable insight about binding

397 cooperativity and fractional accessibility to binding sites may not be gained. Combining models

398 affords another layer of mechanistic insight. As with any model, understanding the assumptions

399 and limitations is vital to interpretation of the underlying mechanisms.

400 In addition, the trend in association constants with respect to physicochemical properties

401 suggests that both hydrophobic and steric effects play a role in degree of binding to serum

402 albumins. As models are developed to predict transport of PFAS through the human body, these

403 intrinsic physicochemical properties are extremely useful. Additional work must be completed in

404 this area, especially for PFAS of different structures than those studied herein.

405

406 ACKNOWLEDGEMENTS

407 This work was funded by the National Institute of Environmental Health Science

408 Sources, Transport, Exposure \& Effects of PFASs (STEEP) Superfund Research Program under

409 grant P42ES027706. STEEP is a partnership of the University of Rhode Island, the Harvard T.H.

410 Chan School of Public Health, and the Silent Spring Institute. 


\section{REFERENCES}

412 Ahrens, L., Bundschuh, M., 2014. Fate and effects of poly- and perfluoroalkyl substances in the 413 aquatic environment: A review. Environ. Toxicol. Chem. 33, 1921-1929.

$414 \quad$ https://doi.org/10.1002/etc.2663

415 Allendorf, F., Berger, U., Goss, K.U., Ulrich, N., 2019. Partition coefficients of four

416 perfluoroalkyl acid alternatives between bovine serum albumin (BSA) and water in

417 comparison to ten classical perfluoroalkyl acids. Environ. Sci. Process. Impacts 21, 1852-

418 1863. https://doi.org/10.1039/c9em00290a

419 Bischel, H.N., MacManus-Spencer, L.A., Luthy, R.G., 2010. Noncovalent Interactions of Long-

420 Chain Perfluoroalkyl Acids with Serum Albumin. Environ. Sci. Technol. 44, 5263-5269.

$421 \quad$ https://doi.org/10.1021/es101334s

422 Bischel, H.N., MacManus-Spencer, L.A., Zhang, C., Luthy, R.G., 2011. Strong associations of

423 short-chain perfluoroalkyl acids with serum albumin and investigation of binding

424 mechanisms. Environ. Toxicol. Chem. 30, 2423-2430. https://doi.org/10.1002/etc.647

425 Boston, C.M., Banacos, N., Heiger-Bernays, W., 2019. Per- and Polyfluoroalkyl Substances: A

426 National Priority for Safe Drinking Water. Public Health Rep. 134, 112-117.

427 https://doi.org/10.1177/0033354919826567

428 Cardenas, A., Gold, D.R., Hauser, R., Kleinman, K.P., Hivert, M.-F., Calafat, A.M., Ye, X.,

429 Webster, T.F., Horton, E.S., Oken, E., 2017. Plasma Concentrations of Per- and

430 Polyfluoroalkyl Substances at Baseline and Associations with Glycemic Indicators and

431 Diabetes Incidence among High-Risk Adults in the Diabetes Prevention Program Trial.

432 Environ. Health Perspect. 125, 107001. https://doi.org/10.1289/EHP1612

433 Chen, H., He, P., Rao, H., Wang, F., Liu, H., Yao, J., 2015. Systematic investigation of the toxic 
mechanism of PFOA and PFOS on bovine serum albumin by spectroscopic and molecular modeling. Chemosphere 129, 217-224. https://doi.org/10.1016/j.chemosphere.2014.11.040

Chen, Y.M., Guo, L.H., 2009. Fluorescence study on site-specific binding of perfluoroalkyl acids to human serum albumin. Arch. Toxicol. 83, 255-261. https://doi.org/10.1007/s00204-0080359-x

Cheng, W., Ng, C.A., 2018. Predicting Relative Protein Affinity of Novel Per- and Polyfluoroalkyl Substances (PFASs) by An Efficient Molecular Dynamics Approach.

Dassuncao, C., Hu, X.C., Nielsen, F., Weihe, P., Grandjean, P., Sunderland, E.M., 2018. Shifting Global Exposures to Poly- and Perfluoroalkyl Substances (PFASs) Evident in Longitudinal

Birth Cohorts from a Seafood-Consuming Population. Environ. Sci. Technol. 52, 3738Environ. Sci. Technol. 52, 7972-7980. https://doi.org/10.1021/acs.est.8b01268 3747. https://doi.org/10.1021/acs.est.7b06044

Domingo, J.L., Nadal, M., 2017. Per- and polyfluoroalkyl substances (PFASs) in food and human dietary intake: A review of the recent scientific literature. J. Agric. Food Chem. 65, 533-543. https://doi.org/10.1021/acs.jafc.6b04683

Eftink, M.R., Ghiron, C.A., 1981. Fluorescence quenching studies with proteins. Anal. Biochem. 114, 199-227. https://doi.org/10.1016/0003-2697(81)90474-7

Fedorenko, M., Alesio, J., Fedorenko, A., Slitt, A., Bothun, G.D., 2021. Dominant entropic

Forsthuber, M., Kaiser, A.M., Granitzer, S., Hassl, I., Hengstschläger, M., Stangl, H., Gundacker, C., 2020. Albumin is the major carrier protein for PFOS, PFOA, PFHxS, PFNA 456 and PFDA in human plasma. Environ. Int. 137, 105324. 
https://doi.org/10.1016/j.envint.2019.105324

458

459

460

461

462

463

464

465

466

467

468

469

470

471

472

473

474

475

476

477

478

479

Gao, K., Zhuang, T., Liu, X., Fu, Jianjie, Zhang, J., Fu, Jie, Wang, L., Zhang, A., Liang, Y., Song, M., Jiang, G., 2019. Prenatal Exposure to Per- and Polyfluoroalkyl Substances (PFASs) and Association between the Placental Transfer Efficiencies and Dissociation Constant of Serum Proteins-PFAS Complexes. Environ. Sci. Technol. 53, 6529-6538. https://doi.org/10.1021/acs.est.9b00715

Gitlin, D., Klinenberg, J.R., Hughes, W.L., 1958. Site of Catabolism of Serum Albumin. Nature $181,1064-1065$.

Grandjean, P., Budtz-Jørgensen, E., 2013. Immunotoxicity of perfluorinated alkylates: calculation of benchmark doses based on serum concentrations in children. Environ. Heal. 12, 35. https://doi.org/10.1186/1476-069X-12-35

Hansch, C., Klein, T.E., 1986. Molecular graphics and QSAR in the study of enzyme-ligand interactions. On the definition of bioreceptors. Acc. Chem. Res. 19, 392-400. https://doi.org/10.1021/ar00132a003

Hartman, T.J., Calafat, A.M., Holmes, A.K., Marcus, M., Northstone, K., Flanders, W.D., Kato, K., Taylor, E. V., 2017. Prenatal Exposure to Perfluoroalkyl Substances and Body Fatness in Girls. Child. Obes. 13, 222-230. https://doi.org/10.1089/chi.2016.0126

Hebert, P.C., MacManus-Spencer, L.A., 2010. Development of a Fluorescence Model for the Binding of Medium- to Long-Chain Perfluoroalkyl Acids to Human Serum Albumin Through a Mechanistic Evaluation of Spectroscopic Evidence. Anal. Chem. 82, 6463-6471. https://doi.org/10.1021/ac100721e

Hu, X.C., Andrews, D.Q., Lindstrom, A.B., Bruton, T.A., Schaider, L.A., Grandjean, P., Lohmann, R., Carignan, C.C., Blum, A., Balan, S.A., Higgins, C.P., Sunderland, E.M., 
2016. Detection of Poly- and Perfluoroalkyl Substances (PFASs) in U.S. Drinking Water Linked to Industrial Sites, Military Fire Training Areas, and Wastewater Treatment Plants. Environ. Sci. Technol. Lett. 3, 344-350. https://doi.org/10.1021/acs.estlett.6b00260

483

484

485

486

487

488

489

490

491

492

493

494

495

496

497

498

499

500

501

502

Lakowicz, J.R., 2006. Principles of fluorescence spectroscopy, 3rd Edition, Joseph R. Lakowicz, editor, Principles of fluorescence spectroscopy, Springer, New York, USA, 3rd edn, 2006. https://doi.org/10.1007/978-0-387-46312-4

Li, L., Song, G.W., Xu, Z.S., 2010. Study on the interaction between bovine serum albumin and potassium perfluoro octane sulfonate. J. Dispers. Sci. Technol. 31, 1547-1551. https://doi.org/10.1080/01932690903294139

Liu, Y., Cao, Z., Zong, W., Liu, R., 2017. Interaction rule and mechanism of perfluoroalkyl sulfonates containing different carbon chains with human serum albumin. RSC Adv. 7, 24781-24788. https://doi.org/10.1039/c7ra02963b

Macmanus-spencer, L. a, Tse, M.L., Hebert, P.C., Bischel, H.N., Luthy, R.G., 2010. Binding of Perfluorocarboxylates to Serum Albumin : A Comparison of Analytical Methods quantitative information about PFCA - albumin interac-. Anal. Chem. 82, 974-981.

MacManus-Spencer, L.A., Tse, M.L., Hebert, P.C., Bischel, H.N., Luthy, R.G., 2010. Binding of Perfluorocarboxylates to Serum Albumin: A Comparison of Analytical Methods. Anal. Chem. 82, 974-981. https://doi.org/10.1021/ac902238u

Ng, C.A., Hungerbühler, K., 2014. Bioaccumulation of perfluorinated alkyl acids: Observations and models. Environ. Sci. Technol. 48, 4637-4648. https://doi.org/10.1021/es404008g

Qin, P., Liu, R., Pan, X., Fang, X., Mou, Y., 2010. Impact of Carbon Chain Length on Binding of Perfluoroalkyl Acids to Bovine Serum Albumin Determined by Spectroscopic Methods. J. Agric. Food Chem. 58, 5561-5567. https://doi.org/10.1021/jf100412q 
503 Ross, I., McDonough, J., Miles, J., Storch, P., Thelakkat Kochunarayanan, P., Kalve, E., Hurst,

504 J., S. Dasgupta, S., Burdick, J., 2018. A review of emerging technologies for remediation of

505 PFASs. Remediat. J. 28, 101-126. https://doi.org/10.1002/rem.21553

506 Salvalaglio, M., Muscionico, I., Cavallotti, C., 2010. Determination of Energies and Sites of

507 Binding of PFOA and PFOS to Human Serum Albumin. J. Phys. Chem. B 114, 14860-

508 14874. https://doi.org/10.1021/jp106584b

509 Steinhardt, J., Krijn, J., Leidy, J.G., 1971. Differences between bovine and human serum

510 albumins. Binding isotherms, optical rotatory dispersion, viscosity, hydrogen ion titration,

511 and fluorescence effects. Biochemistry 10, 4005-4015. https://doi.org/10.1021/bi00798a001

512 Vivian, J.T., Callis, P.R., 2001. Mechanisms of Tryptophan Fluorescence Shifts in Proteins.

513 Biophys. J. 80, 2093-2109. https://doi.org/10.1016/S0006-3495(01)76183-8

514 Waters, N.J., Jones, R., Williams, G., Sohal, B., 2008. Validation of a Rapid Equilibrium

515 Dialysis Approach for the Measurement of Plasma Protein Binding. J. Pharm. Sci. 97,

516 4586-4595. https://doi.org/10.1002/jps.21317

517 Wu, L.-L., Gao, H.-W., Gao, N.-Y., Chen, F.-F., Chen, L., 2009. Interaction of perfluorooctanoic

518 acid with human serum albumin. BMC Struct. Biol. 9, 31. https://doi.org/10.1186/1472-

$519 \quad 6807-9-31$

520 Xiao, F., Sasi, P.C., Yao, B., Kubátová, A., Golovko, S.A., Golovko, M.Y., Soli, D., 2020.

521 Thermal Stability and Decomposition of Perfluoroalkyl Substances on Spent Granular

522 Activated Carbon. Environ. Sci. Technol. Lett. 7, 343-350.

523 https://doi.org/10.1021/acs.estlett.0c00114

524

525 
527 Critical new insights into the binding of poly- and perfluoroalkyl substances (PFAS) to

$532{ }^{\ddagger}$ Department of Biomedical and Pharmaceutical Sciences, University of Rhode Island, Kingston, Rhode Island 02881, United States.

\section{METHODS}

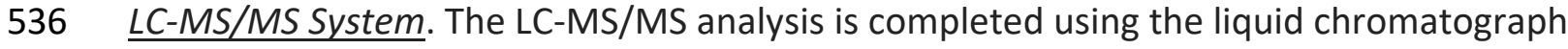

537 (Shimadzu) equipped with a C18 column ( $3 \mu \mathrm{m}, 2.1 \mathrm{~mm} \times 150 \mathrm{~mm}$; Phenomenex) coupled to 538 mass spectrometer (Shimadzu) operating in negative mode.

539

540 LC Conditions. For the analysis, $10 \mu \mathrm{L}$ of dialysate is injected on the $\mathrm{LC}$ column $\left(40^{\circ} \mathrm{C}\right)$ and target

541 compounds are eluted with mobile phase gradient (Table S1.1) consisted of $2 \mathrm{mM}$ ammonium

542 acetate in water and acetonitrile (flow rate $0.5 \mathrm{~mL} / \mathrm{min}$ ).

544 Table S1.1. Gradient Mobile Phase Program.

\begin{tabular}{ccc}
\hline $\begin{array}{c}\text { mobile phase / time } \\
(\mathbf{m i n})\end{array}$ & $\begin{array}{c}\mathbf{A}(\%)-\mathbf{2 m M} \text { ammonium } \\
\text { acetate in water }\end{array}$ & $\begin{array}{c}\text { B (\%) - 2mM ammonium } \\
\text { acetate in acetonitrile }\end{array}$ \\
\hline 0.0 & 50 & 50 \\
6.0 & 15 & 85 \\
8.0 & 15 & 85
\end{tabular}




$\begin{array}{lll}8.01 & 50 & 50 \\ 10.0 & 50 & 50\end{array}$

$545 \quad$ MS/MS Conditions. The triple quadrupole tandem mass spectrometer is operated in multiple

546 reaction monitoring (MRM) mode using negative electrospray ionization (ESI-). Instrument

547 parameters are shown in Table S1.2 and compound specific MS/MS parameters are

548 summarized in S1.3. Values for collision energy are determined through instrument

549 optimization.

550

551 Table S1.2. Mass spectrometer (MS) instrumental parameters.

\begin{tabular}{lc}
\hline Curtain Gas (CUR) & 30 \\
Collision Gas (CAD) & medium \\
IonSpray Voltage (IS) & $-4500 \mathrm{~V}$ \\
Temperature (TEM) & $400^{\circ} \mathrm{C}$ \\
Ion Source Gas 1 (GS1) & 30 \\
Ion Source Gas 2 (GS2) & 30 \\
\hline
\end{tabular}

552

553 Table S1.3 MS/MS Parameters of target PFAS.

\begin{tabular}{llcc}
\hline Analyte & Type & MRM transitions & Collision Energy (V) \\
\hline \hline PFOA & Target & $413 \rightarrow 369 ; 169$ & $11 ; 19$ \\
PFNA & Target & $463 \rightarrow 419 ; 219$ & $10 ; 16$ \\
PFDA & Target & $513 \rightarrow 469 ; 219$ & $11 ; 20$ \\
PFBS & Target & $299 \rightarrow 80 ; 99$ & $34 ; 28$ \\
PFHxS & Target & $399 \rightarrow 80 ; 99$ & $49 ; 43$ \\
PFOS & Target & $499 \rightarrow 80 ; 99$ & $50 ; 44$ \\
Gen X & Target & $347 \rightarrow 169 ; 285$ & $12 ; 20$ \\
\hline
\end{tabular}

Final PFAS concentrations from the dialysis experiment were determined by comparing 556 to external standards of concentrations from $0.02 \mathrm{nM}$ to $200 \mathrm{nM}$. All calibration curves had $\mathrm{R}^{2}$ 
557 values above 0.99 . The value of $K_{a}$ was calculated as $[P L] /([P] x[L])$ with $P$ being protein and $L$

558 being ligand, as calculated in previous works (Allendorf et al., 2019). For all PFAS other than

559 Gen X and PFBS, $K_{a}$ was calculated by using the lowest PFAS to BSA ratio (0.1:1). In the cases of

560 Gen X and PFBS, the small difference between initial and final concentration for this ratio led to

561 the use of a ratio of 2:1 for more precise values.

562

563 RESULTS AND DISCUSSION

564

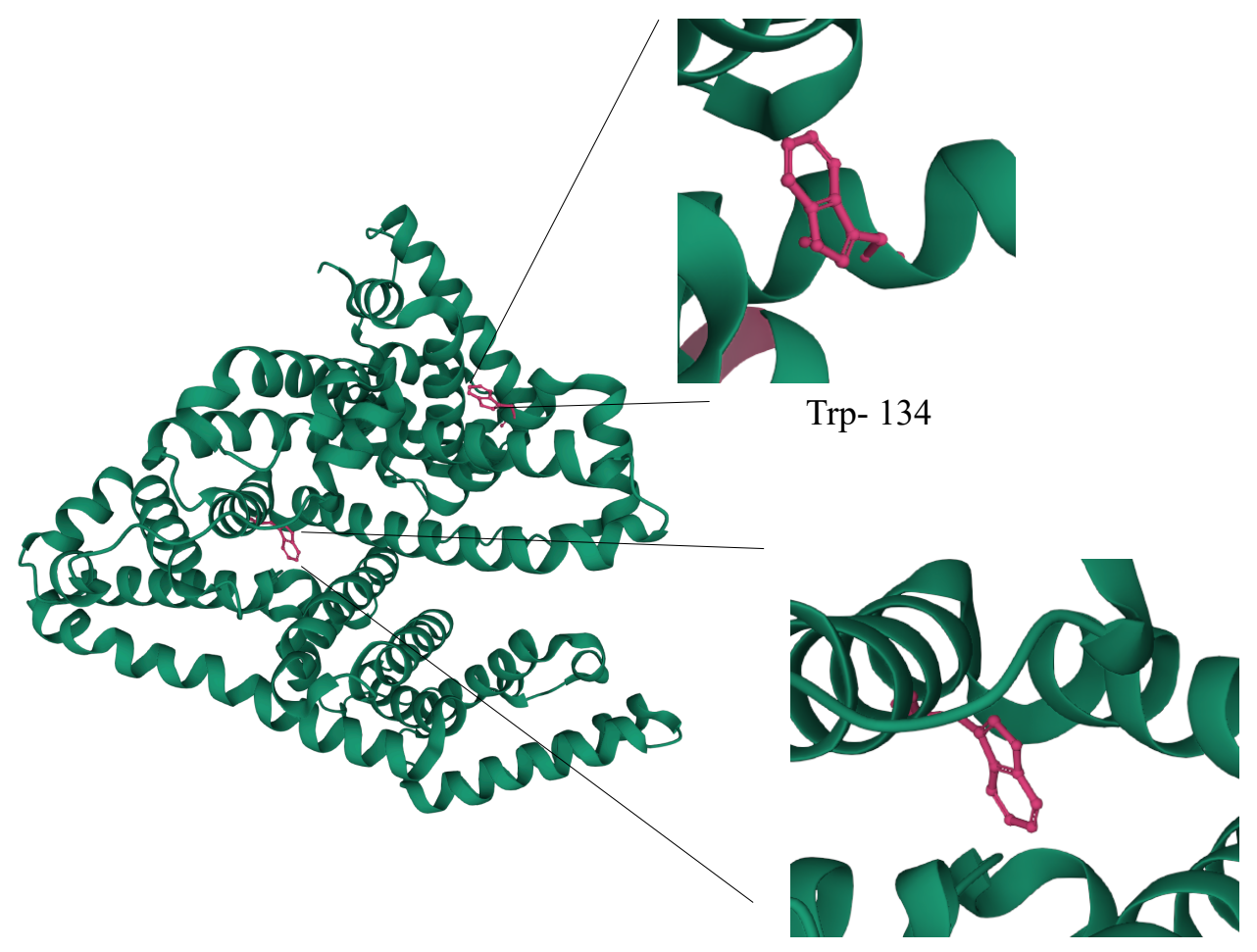

Trp- 213

565 Figure S1. The structure of bovine serum albumin (BSA) with tryptophan residues shown in pink

566 (Protein Data Bank Entry 3V03; Berman et al., 2002; Majorek et al., 2012; Sehnal et al., 2018).

567 Trp-134 is more solvent-accessible while Trp-213 is located within the hydrophobic pocket of

568 the protein. 


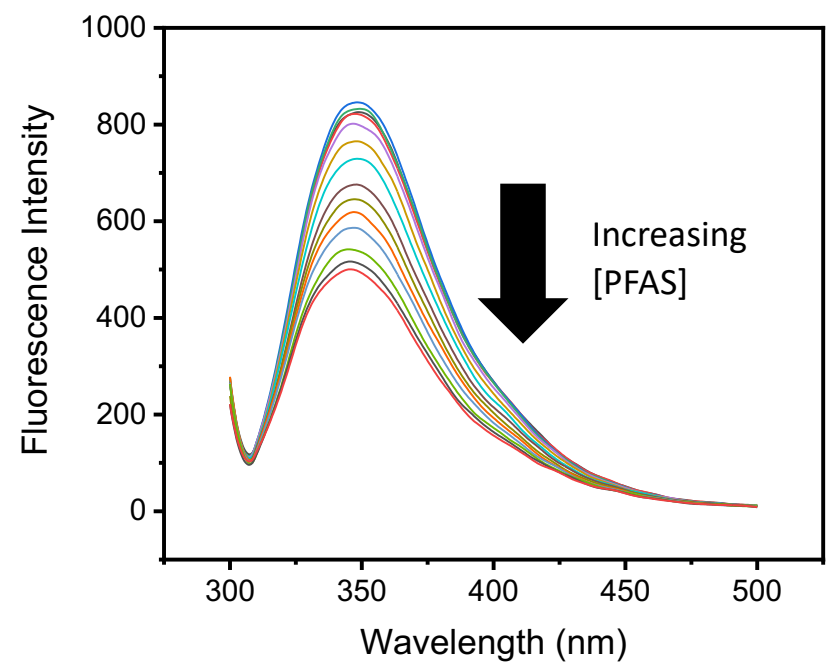

570 Figure S2. Representative fluorescence quenching plot for PFOA $(0-6.8 \mu \mathrm{M})$ added to a BSA

571 concentration of $1.25 \mu \mathrm{M}$ at a temperature of $25^{\circ} \mathrm{C}$. As the concentration of PFAS increases, the

572 fluorescence of BSA decreases, indicative of binding.

573

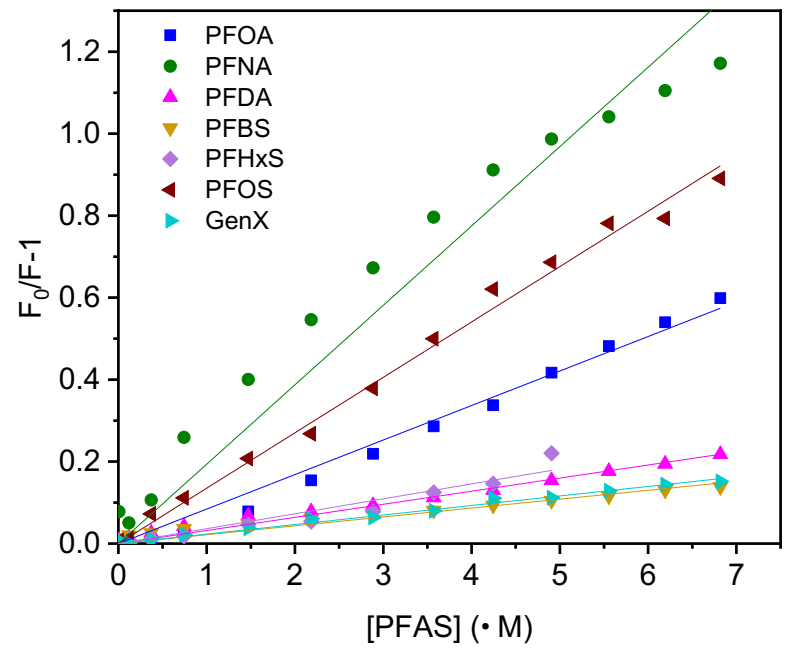

574 Figure S3. Representative Stern-Volmer plot with PFAS concentration ranging from 0 to $6.8 \mu \mathrm{M}$

575 and a BSA concentration of $1.25 \mu \mathrm{M}$ at a temperature of $25^{\circ} \mathrm{C}$. Values of $\mathrm{R}^{2}$ were above 0.97 for 
576 all PFAS other than PFBS at all temperatures studied. Values of $\mathrm{R}^{2}$ for PFBS ranged from $0.92-$

$577 \quad 0.99$.

578

579 Table S2. Stern-Volmer Association Constants (Ksv) from fluorescence quenching experiments,

$580 \quad[$ PFAS $]=0-6.8 \mu \mathrm{M},[\mathrm{BSA}]=1.25 \mu \mathrm{M}$.

\begin{tabular}{lcccc}
\hline PFAS & $\begin{array}{c}\mathrm{K}_{s v}\left(10^{5} \mathrm{M}^{-1}\right) \\
25^{\circ} \mathrm{C}\end{array}$ & $\begin{array}{c}\mathrm{K}_{s v}\left(10^{5} \mathrm{M}^{-1}\right) \\
30^{\circ} \mathrm{C}\end{array}$ & $\begin{array}{c}\mathrm{K}_{s v}\left(10^{5} \mathrm{M}^{-1}\right) \\
35^{\circ} \mathrm{C}\end{array}$ & $\begin{array}{c}\mathrm{K}_{s v}\left(10^{5} \mathrm{M}^{-1}\right) \\
40^{\circ} \mathrm{C}\end{array}$ \\
\hline PFOA & $0.85(0.02)$ & $1.13(0.1)$ & $1.16(0.04)$ & $1.20(0.1)$ \\
PFNA & $1.78(0.22)$ & $2.04(0.03)$ & $1.85(0.01)$ & $1.79(0.07)$ \\
PFDA & $0.31(0.01)$ & $0.31(0.04)$ & $0.36(0.02)$ & $0.40(0.02)$ \\
PFBS & $0.21(0.01)$ & $0.17(0.02)$ & $0.22(0.03)$ & $0.22(0.06)$ \\
PFHxS & $0.48(0.12)$ & $0.52(0.08)$ & $0.61(0.07)$ & $0.56(0.03)$ \\
PFOS & $1.34(0.02)$ & $1.48(0.02)$ & $1.56(0.11)$ & $1.26(0.03)$ \\
Gen X & $0.24(0.01)$ & $0.24(0.03)$ & $0.22(0.02)$ & $0.26(0.07)$ \\
\hline
\end{tabular}

581

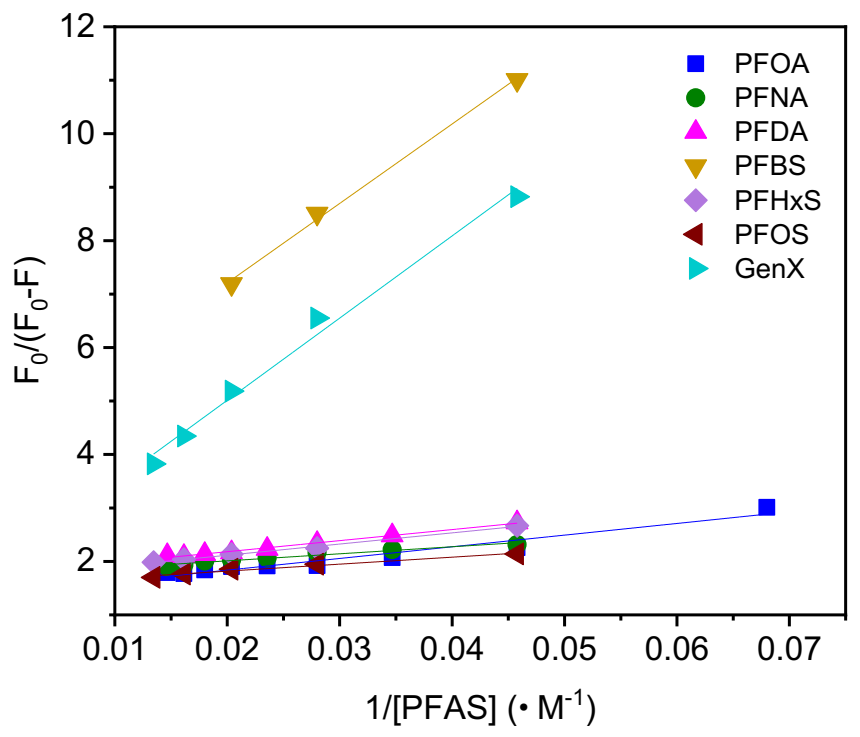

582

583 Figure S4. Representative modified Stern-Volmer plot with PFAS concentration ranging from

58414.7 to $68.2 \mu \mathrm{M}$ for PFCAs and 7.4 to $74.2 \mu \mathrm{M}$ or 35.7 to $74.2 \mu \mathrm{M}$ for PFSAs and a BSA

585 concentration of $1.25 \mu \mathrm{M}$ at a temperature of $25^{\circ} \mathrm{C}$. Values of $\mathrm{R}^{2}$ for all of these plots were 
586 above 0.92 for PFCAs and above 0.95 for PFSAs at all temperatures studied. Inclusion of data in

587 the ranges lower than reported led to deviations from linearity and poor fits.

588

589

590 Table S3. Modified Stern-Volmer Association Constants $\left(K_{M s v}\right)$, fractional accessibility $\left(f_{a}\right)$, Hill

591 association constant $\left(\mathrm{K}_{\text {Hill }}\right)$, and number of Hill binding sites ( $\left.\mathrm{n}_{\text {Hill }}\right)$ from fluorescence quenching

592 experiments, $[\mathrm{PFCA}]=0-68.2 \mu \mathrm{M},[\mathrm{PFSA}]=0-74.2 \mu \mathrm{M},[\mathrm{BSA}]=1.25 \mu \mathrm{M}$. Standard error from

593 duplicate measurements is shown in parenthesis.

\begin{tabular}{|c|c|c|c|c|}
\hline PFAS & $\mathrm{K}_{\mathrm{MSV}}\left(10^{5} \mathrm{M}^{-1}\right)$ & $f_{a}$ & $\mathrm{~K}_{\text {Hill }}\left(10^{5} \mathrm{M}^{-1}\right)$ & $\mathrm{n}_{\text {Hill }}$ \\
\hline \multicolumn{5}{|c|}{$25^{\circ} \mathrm{C}$} \\
\hline PFOA & $1.41(0.18)$ & $0.61(0.02)$ & $1.39(0.38)$ & $1.60(0.06)$ \\
\hline PFNA & $1.37(0.05)$ & $0.62(0.03)$ & $2.55(0.73)$ & $0.96(0.20)$ \\
\hline PFDA & $1.04(0.18)$ & $0.60(0.03)$ & $1.17(0.15)$ & $0.67(0.07)$ \\
\hline PFBS & $0.20(0.08)$ & $0.31(0.08)$ & $0.20(0.05)$ & $0.71(0.04)$ \\
\hline PFHxS & $0.80(0.01)$ & $0.59(0.002)$ & $0.83(0.01)$ & $1.43(0.00)$ \\
\hline PFOS & $1.33(0.14)$ & $0.64(0.001)$ & $1.14(0.21)$ & $1.21(0.03$ \\
\hline Gen X & $0.10(0.02)$ & $0.59(0.07)$ & $0.14(0.02)$ & $1.09(0.13)$ \\
\hline \multicolumn{5}{|c|}{$30^{\circ} \mathrm{C}$} \\
\hline PFOA & $1.88(0.20)$ & $0.58(0.02)$ & $2.37(0.57)$ & 1.49 (0.17) \\
\hline PFNA & $1.44(0.20)$ & $0.63(0.01)$ & $2.78(0.85)$ & $1.10(0.34)$ \\
\hline PFDA & $0.86(0.43)$ & $0.65(0.04)$ & $1.32(0.77)$ & $0.73(0.23)$ \\
\hline PFBS & $0.16(0.02)$ & $0.26(0.03)$ & $0.23(0.06)$ & $0.91(0.05)$ \\
\hline PFHxS & $0.84(0.04)$ & $0.56(0.005)$ & $0.86(0.01)$ & $1.58(0.01)$ \\
\hline PFOS & $1.21(0.05)$ & $0.62(0.01)$ & $1.08(0.02)$ & $1.39(0.14)$ \\
\hline Gen X & $0.09(0.03)$ & $0.67(0.09)$ & $0.13(0.05)$ & $1.19(0.32)$ \\
\hline \multicolumn{5}{|c|}{$35^{\circ} \mathrm{C}$} \\
\hline PFOA & $2.12(0.14)$ & $0.55(0.01)$ & $2.34(0.16)$ & $1.66(0.07)$ \\
\hline PFNA & $1.94(0.17)$ & $0.60(0.04)$ & $3.10(0.87)$ & 1.09 (0.18) \\
\hline PFDA & $1.02(0.57)$ & $0.64(0.07)$ & $1.45(1.40)$ & $0.66(0.16)$ \\
\hline PFBS & $0.25(0.05)$ & $0.26(0.01)$ & $0.15(0.03)$ & $0.73(0.04)$ \\
\hline PFHxS & $1.18(0.10)$ & $0.52(0.01)$ & 1.09 (0.07) & $1.24(0.07)$ \\
\hline PFOS & $1.26(0.02)$ & $0.60(0.01)$ & $1.32(0.11)$ & $1.15(0.04)$ \\
\hline Gen X & $0.17(0.002)$ & $0.46(0.01)$ & $0.16(0.01)$ & $1.06(0.09)$ \\
\hline \multicolumn{5}{|c|}{$40^{\circ} \mathrm{C}$} \\
\hline PFOA & $2.46(0.16)$ & $0.51(0.01)$ & $2.81(0.06)$ & $1.61(0.04)$ \\
\hline
\end{tabular}




\begin{tabular}{lllll} 
PFNA & $1.93(0.06)$ & $0.60(0.01)$ & $4.12(0.17)$ & $0.65(0.01)$ \\
PFDA & $1.59(1.03)$ & $0.55(0.01)$ & $2.74(0.54)$ & $0.58(0.08)$ \\
PFBS & $0.19(0.02)$ & $0.33(0.03)$ & $0.41(0.16)$ & $0.73(0.00)$ \\
PFHxS & $1.17(0.13)$ & $0.52(0.02)$ & $1.29(0.17)$ & $0.96(0.08)$ \\
PFOS & $1.18(0.16)$ & $0.56(0.003)$ & $1.37(0.23)$ & $0.97(0.00)$ \\
Gen X & $0.12(0.01)$ & $0.53(0.06)$ & $0.17(0.06)$ & $1.46(0.62)$ \\
\hline
\end{tabular}

594

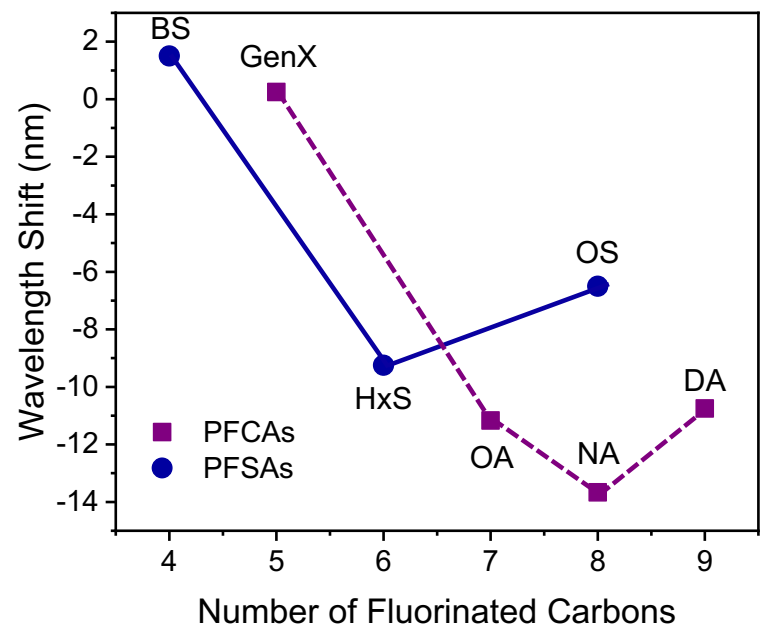

595

Figure S5. A representative plot of the shift in the wavelength of maximum fluorescence

597 emission of BSA at $1.25 \mu \mathrm{M}$ upon addition of PFAS at $68 \mu \mathrm{M}$ at $25^{\circ} \mathrm{C}$. A negative shift in

598 maximum wavelength is indicative of a blue shift in the emission spectrum, which corresponds

599 to the fluorophore experiencing a more hydrophobic environment.

600

601 REFERENCES

602 Allendorf, F., Berger, U., Goss, K.U., Ulrich, N., 2019. Partition coefficients of four perfluoroalkyl 603 acid alternatives between bovine serum albumin (BSA) and water in comparison to ten 604 classical perfluoroalkyl acids. Environ. Sci. Process. Impacts 21, 1852-1863. 
https://doi.org/10.1039/c9em00290a

Berman, H.M., Battistuz, T., Bhat, T.N., Bluhm, W.F., Bourne, P.E., Burkhardt, K., Feng, Z., bank. Acta Crystallogr. Sect. D Biol. Crystallogr. 58, 899-907. https://doi.org/10.1107/S0907444902003451

611 Majorek, K.A., Porebski, P.J., Dayal, A., Zimmerman, M.D., Jablonska, K., Stewart, A.J., Chruszcz, M., Minor, W., 2012. Structural and immunologic characterization of bovine, horse, and rabbit serum albumins. Mol. Immunol. https://doi.org/10.1016/j.molimm.2012.05.011

614 Sehnal, D., A.S. Rose, J. Kovca, S.K. Burley, S. Velankar (2018) Mol*: Towards a common library and tools for web molecular graphics MolVA/EuroVis Proceedings. 